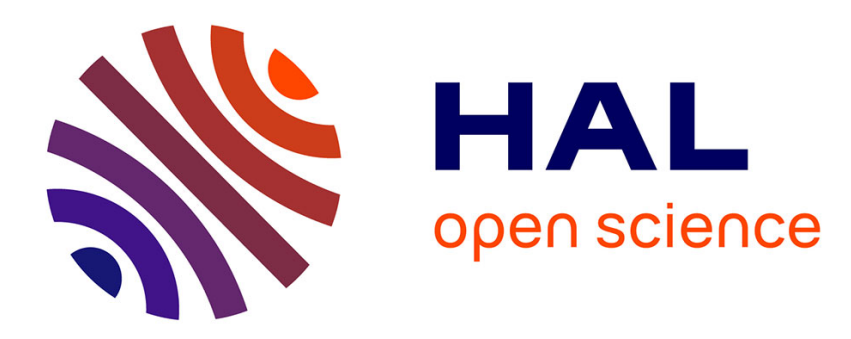

\title{
Canon à électrons à forte brillance utilisant une cathode en borure de lanthane
}

\author{
M. Boussoukaya, A. Septier
}

\section{To cite this version:}

M. Boussoukaya, A. Septier. Canon à électrons à forte brillance utilisant une cathode en borure de lanthane. Revue de Physique Appliquée, 1976, 11 (5), pp.603-607. 10.1051/rphysap:01976001105060300 . jpa-00244095

\section{HAL Id: jpa-00244095 https://hal.science/jpa-00244095}

Submitted on 1 Jan 1976

HAL is a multi-disciplinary open access archive for the deposit and dissemination of scientific research documents, whether they are published or not. The documents may come from teaching and research institutions in France or abroad, or from public or private research centers.
L'archive ouverte pluridisciplinaire HAL, est destinée au dépôt et à la diffusion de documents scientifiques de niveau recherche, publiés ou non, émanant des établissements d'enseignement et de recherche français ou étrangers, des laboratoires publics ou privés. 
Classification

Physics Abstracts

$0.671-2.820$

\title{
CANON A ÉLECTRONS A FORTE BRILLANCE UTILISANT UNE CATHODE EN BORURE DE LANTHANE
}

\author{
M. BOUSSOUKAYA et A. SEPTIER
}

Institut d'Electronique Fondamentale (*), Bâtiment 220, Université Paris XI, 91405 Orsay, France

(Reçu le 8 mars 1976, révisé le 7 mai 1976, accepté le 18 mai 1976)

\begin{abstract}
Résumé. - Un canon à électrons associé à une lentille électrostatique à 3 électrodes, capable de fournir un courant de quelques $\mathrm{mA}$ et un spot de $1 \mathrm{~mm}$ de diamètre à $1 \mathrm{~m}$ de la cathode a été étudié Le canon utilise une cathode en borure de lanthane. La méthode de mesure de la brillance du faisceau est décrite. A tension d'accélération égale, la brillance obtenue est au minimum 100 fois supérieure à celle des canons à cathode de tungstène.
\end{abstract}

Abstract. - An electron source using a $\mathrm{LaB}_{6}$ cathode is investigated. Results and methods of measuring current beam intensity and brightness of $\mathrm{LaB}_{6}$ are given in this paper. Comparison is made between our results and results obtained by others authors using $\mathrm{W}$ cathodes.

1. Introduction. - Pour réaliser un faisceau électronique de très faible diamètre transportant une densité de courant importante, on peut utiliser un canon à électrons muni d'une cathode en borure de lanthane $[1,2,3]$.

Ce type de cathode permet d'obtenir des densités de courant plus importantes [4] que celles des cathodes en tungstène (Fig. 1). Par suite de la faible valeur du travail de sortie du matériau, voisin de $2,6 \mathrm{eV}$, la température d'utilisation reste comprise entre 1200 et $2000{ }^{\circ} \mathrm{C}$. De plus, cette cathode peut fonctionner en ultra-vide et ne craint pas les remises à l'air.

Dans cette étude, nous nous intéresserons particulièrement à un canon débitant 1 à $2 \mathrm{~mA}$ et fournissant à un mètre de la cathode un faisceau de $1 \mathrm{~mm}$ de diamètre, avec une ouverture angulaire de quelques $10^{-3} \mathrm{rad}$.

2. Production du faisceau. -2.1 Description dU CANON. - Un canon à électrons de type triode à cathode émissive plane en borure de lanthane massif a été réalisé sur le modèle de la figure 2.

La cathode est usinée dans un barreau cylindrique de $\mathrm{LaB}_{6}$ de $2 \mathrm{~mm}$ de diamètre. La partie émissive est réduite à une face plane de $0,5 \mathrm{~mm}$ de diamètre $\left(S \simeq 0,20 \mathrm{~mm}^{2}\right)$. Elle est chauffée par bombardement électronique à l'aide d'un filament de tungstène (diamètre $0,2 \mathrm{~mm}$ ) bobiné en forme d'hélice $(5$ spires, diamètre $4 \mathrm{~mm}$, longueur $5 \mathrm{~mm}$ ) et enfermé dans un écran thermique en feuille mince de tantale bien poli d'épaisseur $0,1 \mathrm{~mm}$, qui sert également à repousser vers la tige de $\mathrm{LaB}_{6}$ les électrons émis par le filament.

(*) Laboratoire associé au C. N. R.S.

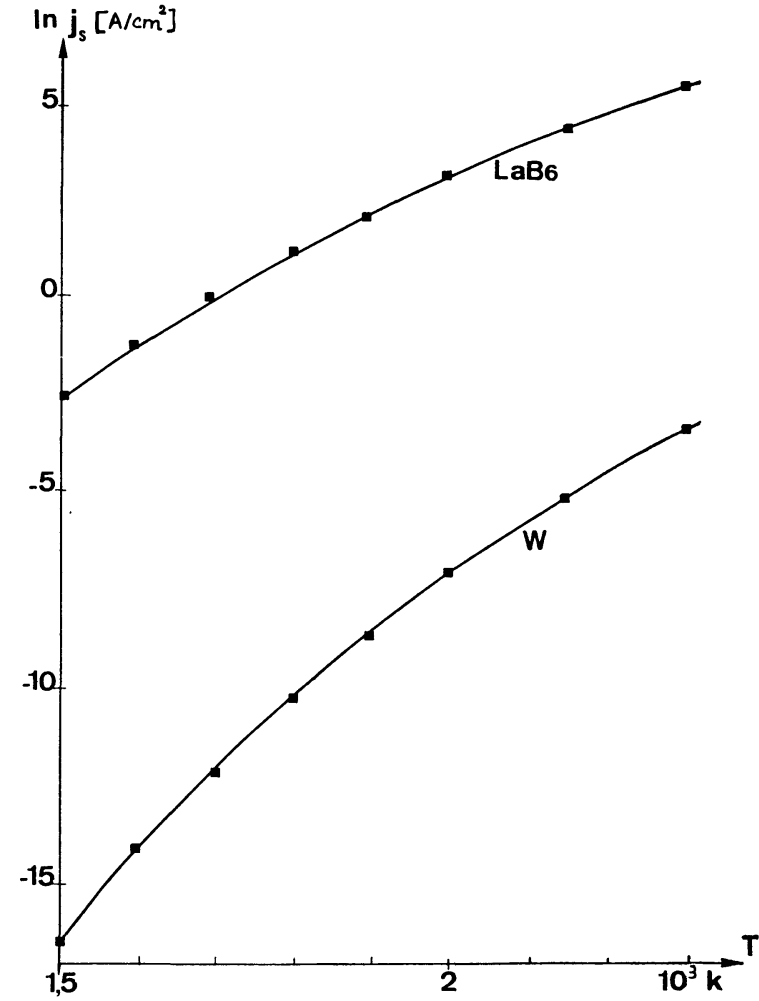

Fig. 1. - Densité de courant de saturation en fonction de la température pour le $\mathrm{W}$ et le $\mathrm{LaB}_{6}$.

L'extrémité de la tige opposée à la face émissive est fixée dans une pièce en cuivre solidaire d'un réservoir refroidi par une circulation de pétrole. Il faut en effet que la température de la région de la tige en contact avec le cuivre reste suffisamment basse pour éviter les 


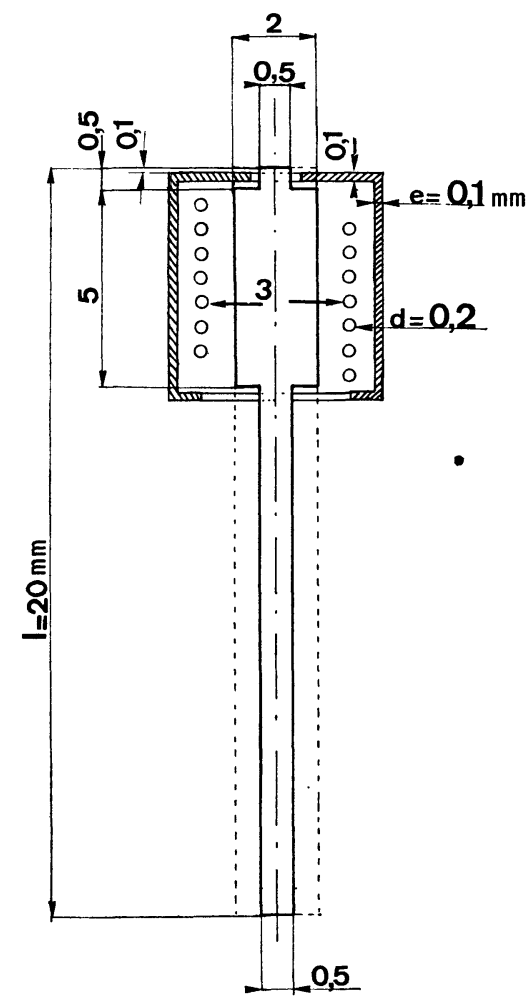

FIG. 2. - Cathode $\mathrm{LaB}_{6}$ et le système de chauffage du canon à électrons.

réactions possibles entre le cuivre et le bore. Le pétrole pur est un excellent isolant, ce qui permet de placer au potentiel de la masse la pompe et le radiateur du circuit de refroidissement ; la connexion avec le canon, porté à une haute tension maximum de $40 \mathrm{kV}$, est assurée par des tubes de téflon.

Pour réduire au maximum la conduction thermique entre la région chauffée à $T=1500^{\circ} \mathrm{C}$ et le support refroidi, le diamètre de la tige a été localement diminué à $0,5 \mathrm{~mm}$ sur une longueur de $15 \mathrm{~mm}$ environ. Un tel rétrécissement, d'après Ahmed et al. [2], permet de réduire par un facteur de l'ordre de 6 la puissance nécessaire au chauffage.

La surface émissive de la tige de $\mathrm{LaB}_{6}$ est située à $0,1 \mathrm{~mm}$ en avant de la surface plane de l'écran en tantale, percée d'un trou de $1 \mathrm{~mm}$ de diamètre. Cet écran est porté à un potentiel légèrement négatif $(6$ à $10 \mathrm{~V})$ par rapport à celui du filament chauffant ; entre filament et cathode, on applique une tension accélératrice réglable de 100 à $250 \mathrm{~V}$. Dans ces conditions, la face avant de l'écran qui entoure la cathode est à un potentiel très négatif par rapport à celui de la surface émissive.

Une deuxième électrode cônique joue le rôle d'un Wehnelt, mais il est nécessaire, dans ce cas particulier, de lui appliquer un potentiel $V_{\mathrm{g}}$ positif par rapport à celui de la cathode, le canon étant naturellement bloqué si $V_{\mathbf{g}}=0$. Comme dans un canon triode classique, le Wehnelt agit à la fois sur le débit du canon et sur la focalisation du faisceau.
Une troisième électrode, réunie à la masse et située à $3 \mathrm{~mm}$ en avant du Wehnelt, accélère les électrons, lorsqu'une tension $V_{0}$, réglable entre 0 et $40 \mathrm{kV}$, est appliquée entre cathode et anode. La figure 3 donne le schéma électrique de l'ensemble du canon.

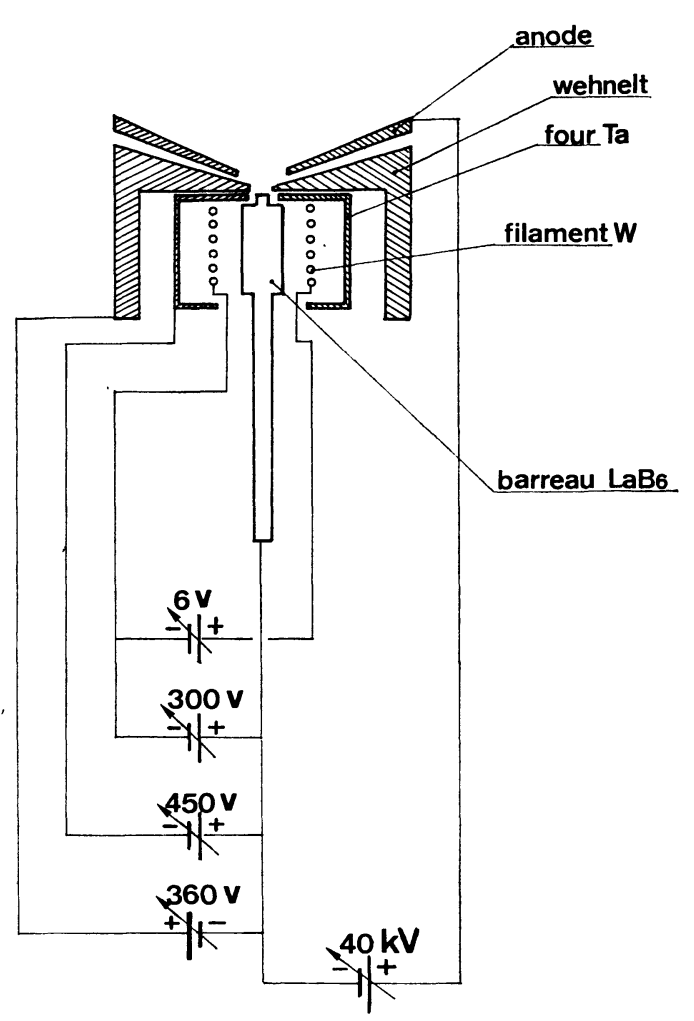

Fig. 3. - Schéma électrique de l'ensemble du canon.

En général, la puissance de chauffage est réglée de telle sorte que le canon puisse travailler en régime de saturation, à la tension d'accélération $V_{0}$ choisie. Le faisceau émis est alors divergent.

Une lentille électrostatique symétrique à trois électrodes, placée immédiatement après l'anode, permet de faire converger les électrons en un cross-over situé à $1 \mathrm{~m} \mathrm{du}$ canon. Pour obtenir un faisceau rectiligne il est nécessaire d'annuler le champ magnétique terrestre sur l'axe du système, par l'emploi de bobinages rectangulaires placés en position de Helmholtz autour de l'enceinte à vide.

\subsection{CARACTÉRISTIQues COURANT-TENSION DU CANON.} - Un courant de saturation de $500 \mu \mathrm{A}$ émis par le barreau en $\mathrm{LaB}_{6}$ a été obtenu sous une tension d'accélération de $12 \mathrm{kV}$ avec une puissance de chauffage de $2,4 \mathrm{~W}(16 \mathrm{~mA} \times 150 \mathrm{~V}$ : courant émis par le filament $\times$ tension de polarisation du filament), ce courant atteint $10 \mathrm{~mA}$ lorsque la puissance est portée à $16 \mathrm{~W}(80 \mathrm{~mA} \times 200 \mathrm{~V})$. Si l'on accroît encore la puissance de chauffage jusqu'à $30 \mathrm{~W}$ par exemple $(120 \mathrm{~mA} \times 250 \mathrm{~V})$, le courant électronique du faisceau peut alors atteindre $10 \mathrm{~mA}$ sous une tension d'accélération très faible. 
Les courbes représentées sur les figures $4 a, 4 b$, illustrent les résultats des mesures du courant émis par le canon en fonction de la tension d'accélération du faisceau et de la puissance de chauffage du barreau.
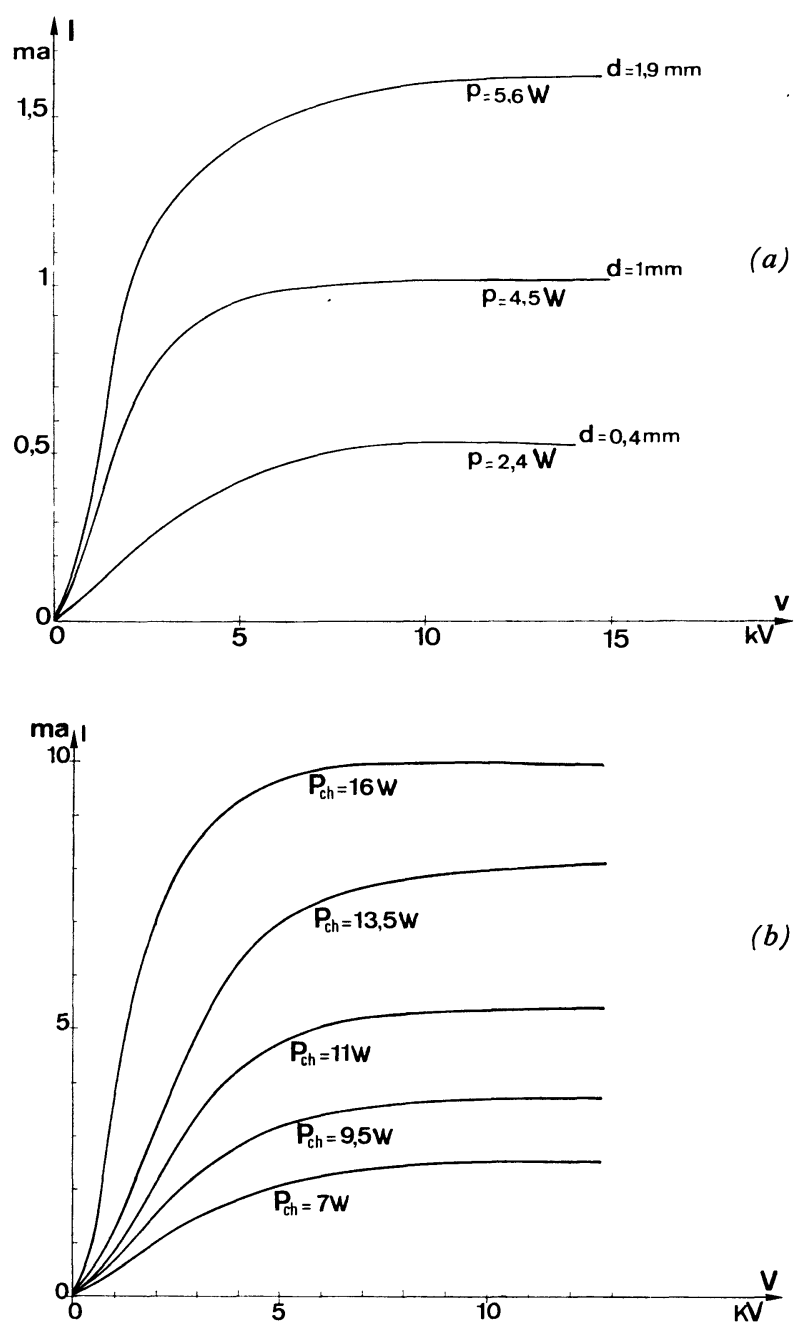

FIG. $4 a, 4 b$. - Caractéristique courant-tension du canon à cathode en $\mathrm{LaB}_{6}$ pour différentes valeurs de la puissance de chauffage.

L'émission suit d'abord un régime de charge d'espace, puis atteint rapidement la saturation. Il est donc possible d'obtenir sous $10 \mathrm{kV}$ un courant de $10 \mathrm{~mA}$ avec une puissance de chauffage de $16 \mathrm{~W}$, ce qui correspond à une densité de courant d'émission de $5 \mathrm{~A} / \mathrm{cm}^{2}$ et une durée de vie de plusieurs centaines d'heures, la température ne dépassant pas $1500^{\circ} \mathrm{C}$ (voir Fig. 1).

La tension positive appliquée au Wehnelt, mesurée par rapport à la cathode était de $220 \mathrm{~V}$.

Sur la figure $4 a$, on s'est limité au domaine des faibles intensités $(0,5$ à $1,5 \mathrm{~mA})$ en cherchant à obtenir à une distance de $1 \mathrm{~m}$ du canon un spot de 1 à $2 \mathrm{~mm}$ de diamètre, grâce à la lentille électrostatique additionnelle. Il suffit de 4,5 W pour obtenir $1 \mathrm{~mA}$, les électrons étant alors entièrement concentrés dans un spot de $1 \mathrm{~mm}$ de diamètre total.
2.3 MeSURE DU DIAMÈTRE DU FAISCEAU. - Le diamètre du faisceau a été déterminé de deux façons différentes :

- Une plaque métallique munie d'un trou de $1 \mathrm{~mm}$ de diamètre a été placée à $1 \mathrm{~m}$ du canon ; elle est suivie d'un collecteur métallique placé à $2 \mathrm{~cm}$ de distance. Ces deux plaques ont été convenablement polarisées pour éviter toute émission secondaire. Pour un réglage convenable des tensions appliquées sur le Wehnelt et sur l'électrode centrale de la lentille, il est possible d'obtenir que tout faisceau électronique d'intensité $I \leqslant 1,15 \mathrm{~mA}$ passe par le trou : aucun courant décelable n'est enregistré sur la première plaque. On mesure ainsi la limite supérieure du diamètre du faisceau - et non, comme c'est généralement le cas - le diamètre à mi-hauteur.

- Une plaque métallique est placée à $1 \mathrm{~m}$ du canon ; elle est percée de deux fentes parallèles de largeur variable $(0 \leqslant l \leqslant 2 \mathrm{~mm})$ de $10 \mathrm{~mm}$ de hauteur et dont les bords voisins sont espacés de $1 \mathrm{~mm}$. Un collecteur polarisé relié à un oscilloscope est placé en arrière des fentes. Le faisceau électronique est déplacé périodiquement sur la plaque grâce à une modulation à $50 \mathrm{~Hz}$ du courant parcourant les bobinages.

La photographie représentée sur la figure 5 donne un exemple du signal obtenu sur le collecteur à partir duquel on peut obtenir la distribution du courant dans le faisceau. Le diamètre du spot, mesuré à la base, est ici inférieur à $1 \mathrm{~mm}$.

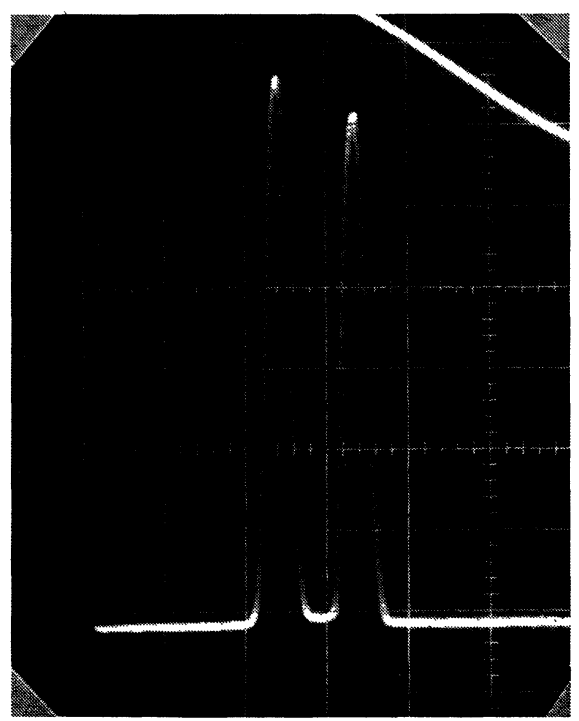

Fig. 5. - Distribution du courant émis par une cathode $\mathrm{LaB}_{6}$ sur un collecteur placé derrière une cible fendue placée à $1 \mathrm{~m}$ du canon.

3. Brillance du faisceau électronique. - 3.1 BRILLANCE : DÉFINITION. - La brillance d'un faisceau électronique de rayon quasi nul (ou brillance axiale) peut être définie par l'expression suivante :

$$
B=\frac{J_{\mathrm{s}} \varepsilon_{\text {cin }}}{\pi k T}
$$


où $J_{\mathrm{s}}$ représente la densité du courant d'émission de la cathode ; $\varepsilon_{\text {cin }}$ l'énergie cinétique longitudinale du faisceau ; $k$ la constante de Boltzmann et $T$ la température en degrés Kelvin. ( $k T$ est alors l'énergie transversale des électrons.)

La formule précédente est utilisée pour calculer la brillance d'un faisceau électronique lorsqu'on suppose une densité d'émission uniforme sur la cathode et des angles d'émission identiques en tous points de la cathode (émittance rectangulaire). Cette brillance se conserve d'un bout à l'autre du système optique, en supposant celui-ci dépourvu d'aberrations, lorsque le faisceau circule dans un espace équipotentiel. Dans ces conditions, on peut remplacer dans (1) $\varepsilon_{\text {cin }}$ et $k T$ par leurs expressions respectives :

$$
\varepsilon_{\mathrm{cin}}=\frac{1}{2} m v_{z}^{2} \quad \text { et } \quad k T=\frac{1}{2} m v_{r \max }^{2}
$$

on trouve alors :

$$
B=-\frac{J_{s}}{\pi\left(\frac{v_{r \max }}{v_{z}}\right)^{2}}=\frac{I}{\pi S \alpha_{\max }^{2}},
$$

soit

$$
B=\frac{I}{\pi^{2} r_{0}^{2} \alpha_{\max }^{2}}
$$

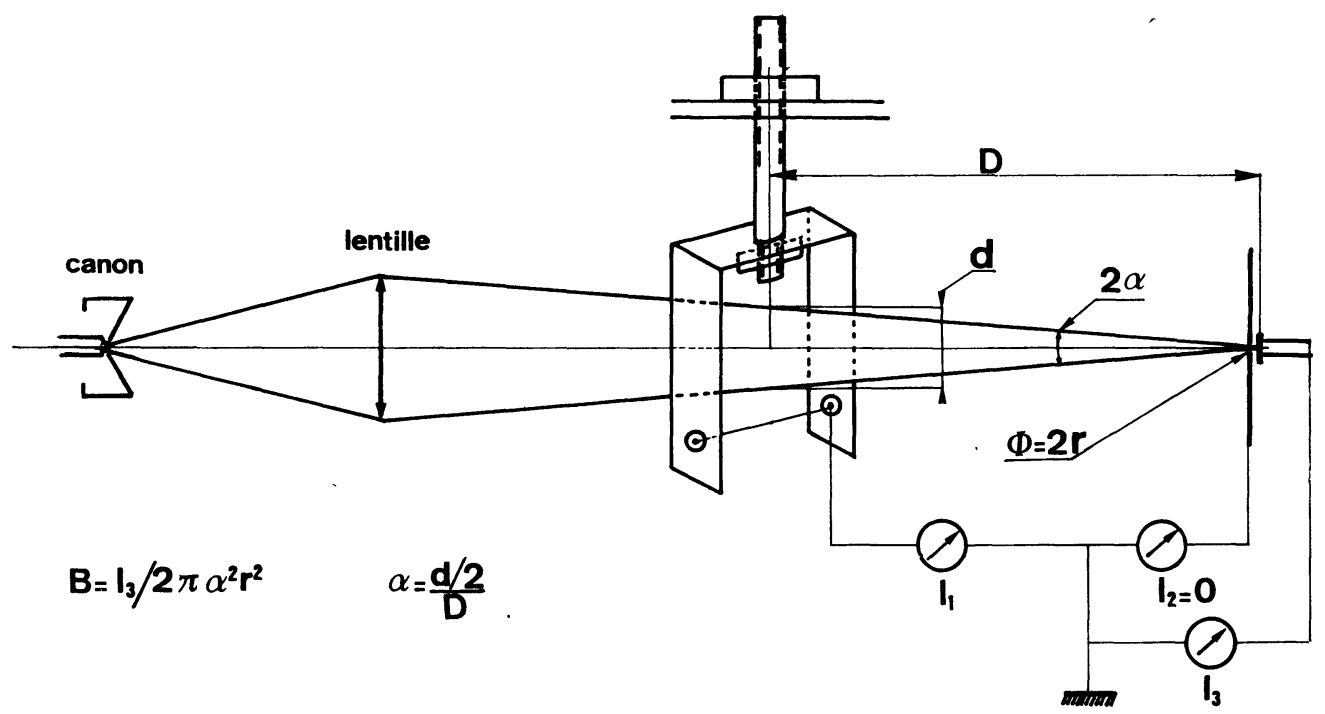

Fig. 6. - Schéma représentant le principe de mesure de l'angle d'ouverture du faisceau au niveau de la cible. où $I=\pi r_{0}^{2} J_{\mathrm{s}}$ représente l'intensité totale du courant électronique considéré ; $r_{0}$ et $\alpha_{\max }=(\mathrm{d} r / \mathrm{d} z)_{\max }$ désignent respectivement le rayon du faisceau et sa demiouverture angulaire au point de mesure de $I$.

Dans notre cas, l'émittance du faisceau est sensiblement elliptique et nous utiliserons l'expression [5] :

$$
B=\frac{2 I}{\pi^{2} r^{2} \alpha_{\max }^{2}} .
$$

3. 2 RÉSULTATS EXPÉRIMENTAUX. - L'angle $\alpha_{\max }$ a été mesuré à $D=55 \mathrm{~cm}$ du collecteur par détermination du diamètre du faisceau en utilisant un fil de cuivre de $0,2 \mathrm{~mm}$ de diamètre fixé sur un étrier relié à un ampéremètre et porté par une tige métallique mobile verticalement. La figure 6 représente le schéma du dispositif expérimental utilisé.

Connaissant $r_{0}, V_{0}$ et $\alpha_{\max }$, au niveau du collecteur, la brillance du faisceau électronique peut être calculée. Les résultats obtenus avec des électrons de $12 \mathrm{kV}$ sont portés sur le tableau I en fonction de l'intensité du faisceau. La variation d'intensité est obtenue en faisant varier la température de la cathode.

Nos résultats sont en bon accord avec ceux obtenus par d'autres auteurs [6] utilisant le même type de cathode.

\section{TABLEAU I}

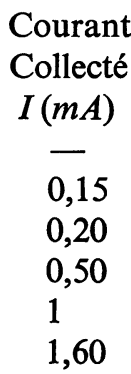

\begin{abstract}
Diamètre du faisceau sur le collecteur

$$
2 r_{0}(\mathrm{~mm})
$$
\end{abstract}

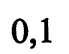

0,18

0,40

1

1,9

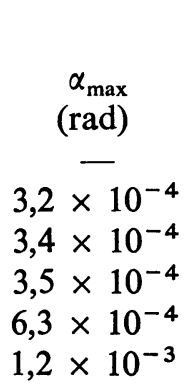

Brillance normalisée $B_{\mathrm{n}}$ $\left(\mathrm{A} \mathrm{cm}^{-2} \mathrm{rad}^{-2}\right)$

$\begin{array}{rr}-\left(\mathrm{A} \mathrm{cm}^{-2} \mathrm{rad}^{-2}\right) & - \\ - & 1,3 \times 10^{8} \\ 2,6 \times 10^{7} & 4,5 \times 10^{7} \\ 10^{6} & 2,1 \times 10^{7} \\ 10^{6} & 2,1 \times 10^{6} \\ 10^{5} & 2,6 \times 10^{5}\end{array}$


Pour des courants de même intensité, la brillance des faisceaux issus de cathodes en $\mathrm{LaB}_{6}$ est nettement supérieure à celles des faisceaux issus de cathodes en tungstène. En effet, pour des faisceaux d'intensité comprise entre 150 et $200 \mu \mathrm{A}$ obtenus à partir d'une cathode de tungstène et accélérés sous des tensions allant jusqu'à $50 \mathrm{kV}$, on obtient en moyenne $[7,8,9]$ des brillances de l'ordre de $2 \times 10^{5} \mathrm{~A} \mathrm{~cm}^{-2} \mathrm{rad}^{-2}$.

Pour mieux comparer ces derniers résultats avec ceux du tableau I, on a introduit la notion de brillance normalisée $B_{n}$, pour tenir compte de l'énergie des électrons, lorsque celle-ci varie toutes choses égales par ailleurs; on montre en effet que seule la brillance normée $B_{\mathrm{n}}$ reste invariante.

$B_{\mathrm{n}}$ est donnée par l'expression :

$$
B_{\mathrm{n}}=\frac{B}{\beta^{2} \gamma^{2}}=\frac{2 I}{\pi^{2} r_{0}^{2} \alpha_{\max }^{2} \beta^{2} \gamma^{2}}
$$

où $\beta=v / c$ représente la vitesse réduite des électrons du faisceau ;

$$
\gamma=\frac{m}{m_{0}}=\frac{1}{\sqrt{1-\beta^{2}}}
$$

est l'accroissement de masse de l'électron accéléré.

La brillance normalisée de faisceaux de faibles intensités $(150$ à $500 \mu \mathrm{A})$ obtenu dans notre canon à cathode de $\mathrm{LaB}_{6}$ est environ 125 fois plus forte que celle de canons triodes classiques à cathode de tungstène, fonctionnant à beaucoup plus haute température $\left(T \sim 2500^{\circ} \mathrm{C}\right)$ et ayant une surface émissive de forme moins bien définie.

Il faut noter cependant - mais ce phénomène est observable dans tous les types de canons - que $B$ décroît lorsque $I$ augmente, alors qu'on devrait observer une croissance linéaire de $B$ avec $I$. Trois facteurs interviennent pour réduire $B$ à tension d'accélération constante :

- L'accroissement des vitesses transversales par suite d'une augmentation de la température de fonctionnement (qui passe de $T=1200^{\circ} \mathrm{C}$ à $T=1450^{\circ} \mathrm{C}$ pour l'exemple fourni sur le tableau I), entraînant l'augmentation de $\alpha_{\max }$;

- l'accroissement du rayon $r_{0}$ du spot et de $\alpha_{\max }$ sous l'influence de la charge d'espace croissante du faisceau ;

- enfin, l'aberration d'ouverture du canon qui croît comme $\left(d_{\max }\right)^{3}$ lorsqu'on désigne par $d_{\max }$ le diamètre maximum du faisceau à l'intérieur des électrodes de la lentille de focalisation.

4. Conclusion. - Un système triode à cathode en $\mathrm{LaB}_{6}$ associé à une lentille unipotentielle a permis d'obtenir un courant supérieur au mA dans un spot électronique de $1 \mathrm{~mm}$ de diamètre situé à un mètre du canon. La brillance mesurée est plus de 100 fois supérieure à celle qu'on obtiendrait avec une cathode de tungstène.

Ce canon a été utilisé dans un système hacheur de faisceau, destiné à la production de paquets d'électrons très courts à une fréquence de $2600 \mathrm{MHz}$, en vue de leur injection dans une cavité accélératrice supraconductrice $[10,11]$.

Remerciements. - Les auteurs remercient $M$. $H$. Mazubert, technicien du C. N. R. S. à l'IEF, pour la fabrication du canon à cathode $\mathrm{LaB}_{6}$ et pour sa participation aux mesures.

\section{Bibliographie}

[1] Broers, A. N., J. Appl. Phys. 38 (1967) 1991-2.

[2] Ahmed, H., Proc. 5th Europ. Congress on Elect. Microscopy, Manchester, 1972.

[3] Aнmed, H. et al., Rev. Sci. Instrum. 43 (1972) 1048.

[4] Ahmed, H. et Boers, A. N., J. Appl. Phys. 43 (1972) 2185.

[5] SEPTIER, A., Production of ion beams of high intensity, Focusing of Charged Particles (édité par Academic Press) 1967, vol. 2, chap. 3, 4, p. 152.

[6] Von Vogt, R., Optik 36 (1972) 262.

[7] Sнimoyama, H. et al., J. Electr. Microscopy 21 (1970) 119.
[8] HAINe, M. et LINDER, D., High brightness electron guns, Focusing of Charged Particles, vol. 1, chap. 2.1, p. 233251 (édité par Academic Press) 1967.

[9] Tanaka, T., J. Phys. C. Solid States 7 (1974) L-177.

[10] Boussoukaya, M., Septier, A. et WartSki, L., Revue Phys. Appl. 11 (1976) 297.

[11] Boussoukaya, M., Dynamique des électrons dans les accélérateurs linéaires supraconducteurs et les déflecteurs hyperfréquences, Thèse de Doctorat d'Etat, Orsay A-1445 (mars 1975). 